NASZA DERMATOLOGIA Online OUR DERMATOLOGY Online

Source of Support: Nil

Competing Interests: None

\section{CLASSICAL FUSIFORM EXCISION OF MELANOCYTIC NEVI: OUR EXPERIENCE}

\author{
Iffat Hassan ${ }^{1}$, Shazia Jeelani ${ }^{1}$, Abid Keen ${ }^{1}$, Mashkoor Wani ${ }^{2}$ \\ ${ }^{I}$ Department of Dermatology, STD and Leprosy, Government Medical College, \\ Srinagar (University of Kashmir), $J \& K$, India \\ ${ }^{2}$ Department of Dermatology, STD and Leprosy, Government Medical College, Jammu \\ (University of Kashmir), $J \& K$, India
}

Corresponding author: Ass. Prof. Iffat Hassan

hassaniffat@gmail.com

\begin{abstract}
Introduction: Facial melanocytic nevi whether acquired or congenital may pose an aesthetic problem for many patients, especially women. There are many methods of removal of melanocytic nevi including surgical and non-surgical. However, surgical excision by classical fusiform excision remains the most widely used and one of the best methods taking all perspectives into consideration.

Aim: To evaluate efficacy of classical fusiform excision for the removal of facial melanocytic nevi.

Methods: In a prospective study, 55 facial melanocytic nevi were removed by fusiform excision technique. Incision was given around the nevus in an elliptical pattern, three times as long as it was wide and along the relaxed skin tension lines. Dissection was carried down to midsubcutaneous tissue to remove the nevus down to its full depth. Wound was closed by simple interrupted sutures using 5-0 prolene with adequate undermining of the wound edges. Sutures were removed on $7^{\text {th }}$ postoperative day.

Results: Complete removal of nevi was achieved in all patients with good to excellent cosmetic results. The scar mark if any, would fade in 3-4 months and were imperceptible in 6-9 months.

Conclusions: Fusiform excision is one of the best, most widely used and time-tested procedure for complete surgical excision of melanocytic nevi.
\end{abstract}

Key words: melanocytic naevi; naevi; fusiform excision

\section{Introduction}

Melanocytic naevi are common lesions that can be found on the integument of almost all individuals. Some patients present with few lesions, while others have hundreds. The number on a given individual increases in rough proportion to the degree of skin pigmentation.

Facial melanocytic naevi whether acquired or congenital may pose an aesthetic problem for many patients especially women. There are many methods of removal of melanocytic naevi including surgical and non-surgical. However, surgical excision by classical fusiform excision remains the most widely used and one of the best methods taking into consideration all perspectives. We have found good cosmetic result and complete removal of naevus with no recurrence while removing the facial naevus by means of fusiform excision and here by report our findings.

\section{Material and Methods}

A prospective study was carried out in the Department of Dermatology, STD \& Leprosy (Associated teaching hospital of Govt. Medical College, Srinagar) from January 2010 to December 2011 involving 50 patients (45 female and 5 male) in the age range of 18-51 years having a total of 55 melanocytic naevi on their face which were excised. In two female patients, multiple facial melanocytic naevi were excised and in all others single naevus was excised. The size of the naevi varied from 1 to $1.5 \mathrm{~cm}$ diameter.

Routine laboratory investigations including complete hemogram, fasting blood sugar, bleeding and clotting time were done in all patients. Xylocaine sensitivity was performed in all patients. Close-up photographs were taken and an informed consent was obtained. The area was cleaned with povidine iodine and spirit. Under local anaesthesia using $2 \%$ lignocaine, incision was given around the naevus in an elliptical pattern using scalpel blade.

The length of the incision being approximately 3 times its width. Care was taken to make the incision always along the relaxed skin tension lines. 
The precise inclination of these lines was confirmed by having the patient engaged in a variety of exaggerated facial expressions and by pinching the skin in between thumb and forefinger. A total of 10 naevi were on the natural facial folds including nasolabial folds, on forehead along the wrinkles in a middle aged woman. These lesions healed with excellent cosmetic results obtained as early as 15 days after the excision.

Dissection was carried down to mid-subcutaneous tissue to remove the naevus down to its full depth. In case of larger naevi on relatively less mobile skin subcutaneous sutures using absorbable Vicryl were given so as to approximate the wound margins. Wound was closed by simple interrupted sutures using non-absorbable polypropylene monofilament suture (5-0 Prolene) and with adequate undermining of the wound edges. Wound was dressed with sterile gauze packs. Systemic antibiotics were prescribed for 1 week post-operation to minimize the chances of post- operative infection. The specimen obtained was sent for histopathological examination in all cases. Sutures were removed on $7^{\text {th }}$ postoperative day. After the suture removal, scar was immediately treated with topical silicone gel to fade out the suture marks.

\section{Results}

Complete removal of naevi was achieved in all patients with good to excellent cosmetic results (Fig. 1a-d, 2a-c, 3ac, $4 \mathrm{a}, \mathrm{b})$. The complications noted during the procedure were minor and included hematoma formation and slight wound infection in 3 patients each and stitch granuloma in one patient. The scar mark, if any faded in 2-3 months and was imperceptible in 6-7 months. No recurrences were noted. Patients were very much satisfied with the result outcome to the extent that patients with multiple facial melanocytic naevi requested for the removal of other facial naevi.

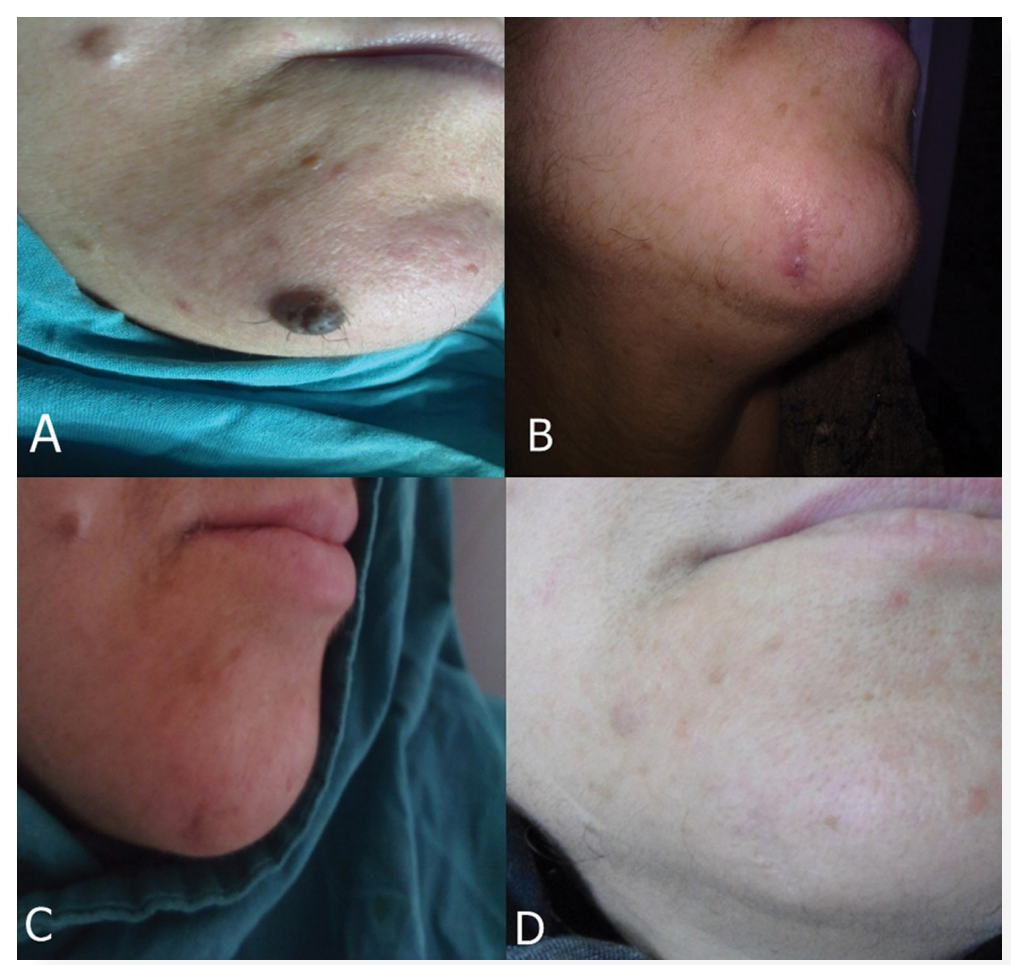

Figure 1. A. large melanocytic hairy nevus on chin of 48 year old; B. weeks post-excision; C. 4 weeks post excision; D. excellent cosmetic result after 12 weeks

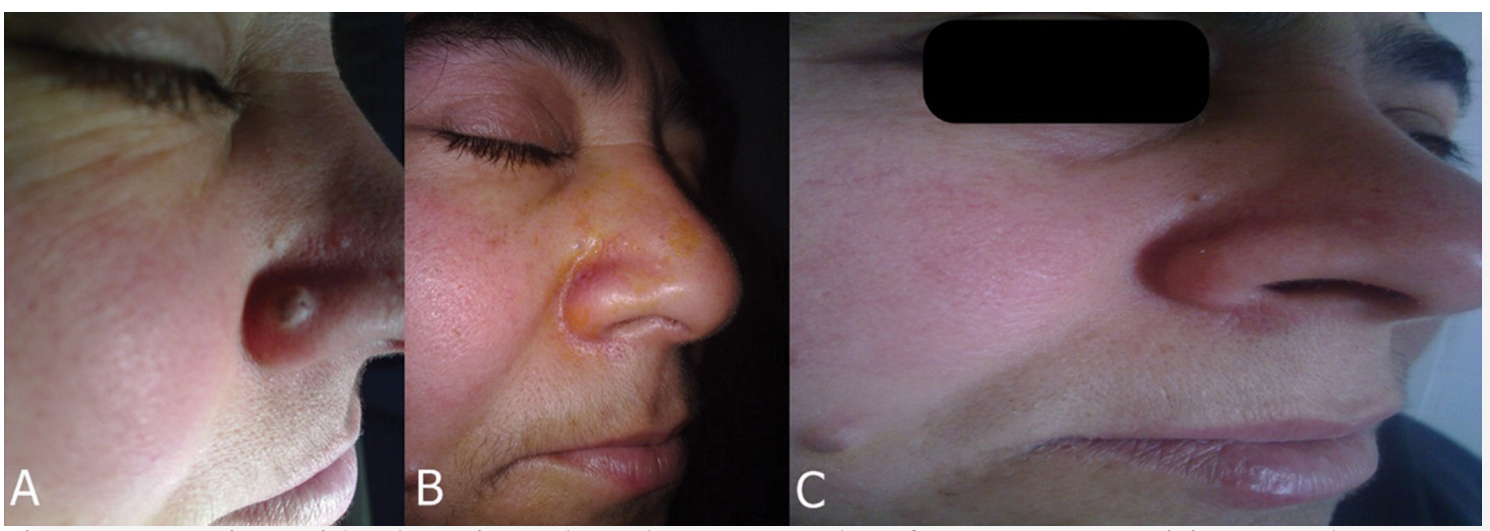

Figure 2. A. patient with a large intra-dermal neavus on alae of nose; B. post excision 2 weeks; C. post excision 4 weeks 


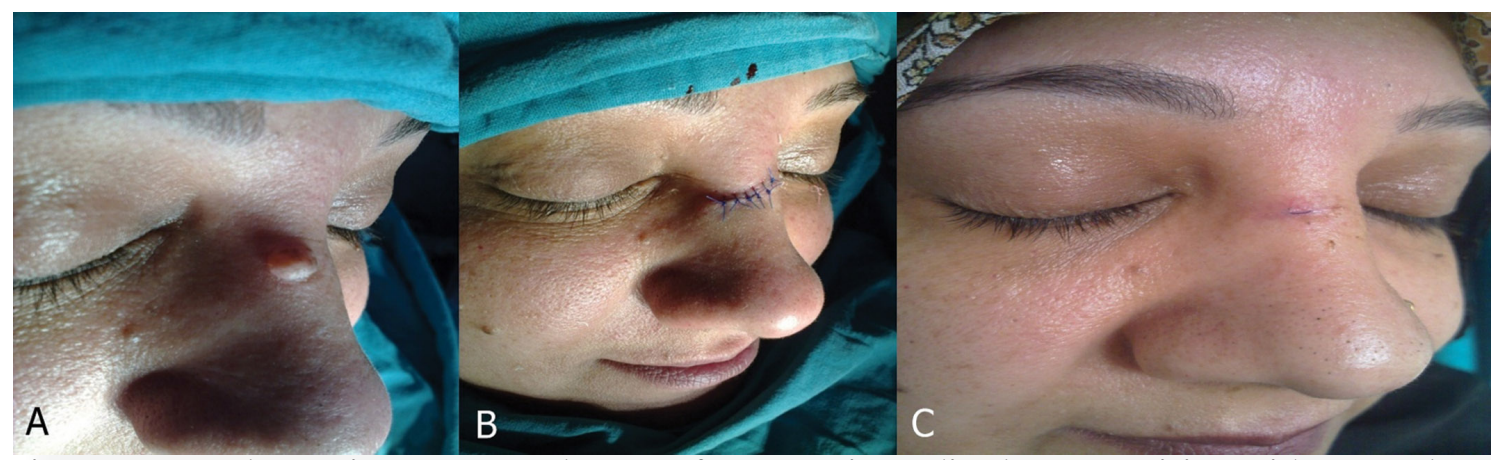

Figure 3. A. melanocytic naevus on dorsum of nose; B. immediately post-excision with 5-0 Prolene sutures; C. same lesion after 2 weeks, subcutaneous Vicryl coming out from the wound which was cut off, with good cosmesis

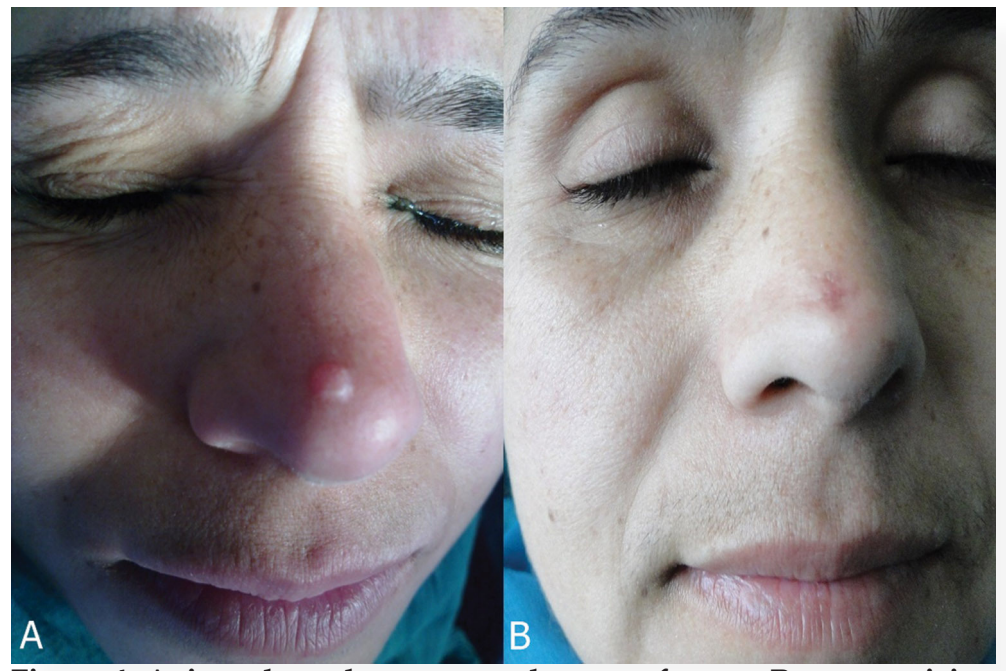

Figure 4. A. intradermal naevus on dorsum of nose; B. post-excision 2 weeks

\section{Discussions}

Along with recent advances in the knowledge of pigment formation and of pigment cell biology, there has been an increasinginterestinthepigmentedmoleornaevus. Melanocytic naevi are normal benign proliferations of melanocytes. Although the risk of a naevus evolving into a melanoma is extremely small, melanocytic naevi are both risk factors for melanoma and precursors of melanoma. The prevalence of pigmented lesions present at birth varies considerably between published series; this is principally because of the ethnic mix of the patients examined, as congenital melanocytic naevi may be more common in black or Asian children [1], but they are generally considered to be present in between 1 and $2 \%$ of newborns [2]. Some patients present with few lesions, while others have hundreds. Melanocytic naevi develop through childhood and twin studies provide good evidence that naevus number is predominantly genetically determined [3] with a smaller effect of sun exposure [4].

Melanocytic naevi can be broadly divided into congenital and acquired types. Congenital melanocytic naevi vary considerably in size and are classified according to American National Institutes of Health (NIH) consensus definition [1] as small $(<1.5 \mathrm{~cm})$, intermediate $(1.5-20 \mathrm{~cm})$, or large/giant $(>20 \mathrm{~cm})$. Conventional or common acquired melanocytic naevi are generally less than $1 \mathrm{~cm}$ in diameter and evenly pigmented.

Not all melanocytic naevi that change are malignant, especially if change is noted in a person younger than 40 years. However, change that is perceptible over a short time is an indicator of potential malignancy and designates a lesion deserving a biopsy. An Australian study found that $16 \%$ of benign lesions changed (as measured by sequential digital dermoscopic imaging) over an interval of 2.5-4.5 months. The proportion of benign lesions that changed was higher in persons aged $0-35$ years than in those aged 36-65 years but rose again in the elderly (age $>65$ years) [5].

Since it is an extremely common lesion, clinically often disfiguring, many patients are seen who desire cosmetic removal of their moles. Removal of a medium size melanocytic naevus whether congenital or acquired over exposed parts, especially over face is warranted for its cosmetic, embarrassment rather than for its potential to cause malignancy.

Several methods of dealing with the common mole are described in the literature. These may be divided into two main types: deep excision, which removes the entire lesion, and other methods, which do not completely remove it. Out of the many methods available (viz, surgical resection, shave excision, laser removal) cosmetic result of surgical resection with primary suturing is always preferable [6]. This is technically less demanding and can be performed even by a novice cutaneous surgeon if basic principles of cosmetic surgery are taken care of.

Melanocytic naevi removed for cosmesis are often removed by tangential or shave excision however such a procedure has its potential disadvantages as there are chances of incomplete removal and subsequent recurrence of the naevus. Moreover, shave excisions can sometimes heal with a permanent scar formation. 
Punch excision can be used for relatively small dome-shaped lesions [7]. However when used for larger lesions, they may lead to the formation of dog ears.

Melanocytic naevi can also be removed by means of $\mathrm{CO} 2$ laser. However there is a high incidence of repigmentation following $\mathrm{CO} 2$ laser treatment of melanocytic naevi [8]. Cost implications of laser treatments should also be considered when choosing treatment options for the removal of melanocytic naevi. Finally, laser-induced malignant transformation of naevus cells remains a theoretical concern but such concerns have not been found to be true in clinical practice [9].

Large lesions may require complete excision with sutured closure, even if known to be benign, because lesions exceeding $1 \mathrm{~cm}$ in diameter often are not amenable to the shave technique.

A simple conservative excisional biopsy with a sutured closure is usually the most expeditious means to diagnosis if concern exists regarding the possibility of melanoma. If the lesion is found to be benign, then, ordinarily, no further treatment is required. Providing the pathologist with a complete excisional specimen affords him or her best opportunity to make an accurate diagnosis because all available criteria (including low-magnification attributes such as size, circumscription and symmetry) can be applied to the lesion.

The elliptical or fusiform, excision is the classic approach to removing an approximately round or linear lesion [10,11]. An excision of this shape is preferred because of the subsequent ease of its closure. A round excision larger than a 4-mm punch is difficult to close without leaving excess outpouchings of skin or dog ears.

The ellipse should be long enough so it can be sutured together without dog ears but no longer than necessary to minimize the length of the inevitable scar. The process begins by marking the lesion to be removed. A permanent marker or other skin marker may be used to circle the target lesion. Often, a free margin of several millimetres must be removed from around the primary lesion, and the mark for this margin can be drawn as a larger concentric circle. Then, an ellipse should be marked around the larger circle. The diameter of the circle should form the short axis of the ellipse, and the longer axis should be 3-4 times this length.

The orientation of the ellipse is important. Ideally, the long axis should lie parallel to the relaxed skin tension lines at the body site involved. Diagrams that display the common skin tension lines on the face and body are available. The precise inclination of these lines can be confirmed by having the patient engage in a variety of exaggerated facial expressions. On the arms and legs, flexion and extension can substitute for such manoeuvres. The surgeon can obtain this information at the surgical site by deliberately wrinkling the skin at the surgical site between his or her thumb and forefinger. By placing the ellipse along relaxed skin tension lines, the surgeon ensures that the final scar is parallel to these lines. If the scar is thin and small and if the patient is elderly, it may be barely noticeable, appearing as merely another wrinkle or crease to the untrained observer.

The most commonly used and versatile suture in cutaneous surgery is the simple interrupted suture [12]. Compared with running sutures, interrupted sutures are easy to place, have greater tensile strength, and have less potential for causing wound oedema and impaired cutaneous circulation. Interrupted sutures also allow the surgeon to make adjustments as needed to properly align wound edges as the wound is sutured. Disadvantages of interrupted sutures include the length of time required for their placement and the greater risk of crosshatched marks (ie, train tracks) across the suture line. The risk of crosshatching can be minimized by removing sutures early to prevent the development of suture tracks.

To sum up, the classical fusiform excision of melanocytic naevi offers certain advantages in the form of a better cosmetic scar, no chances of recurrences, histopathological examination of the lesion, minimal expertise in performing the simple elliptical excisions, and cost-effectiveness of the procedure as compared to repeated laser treatments.

Based on our results, we can conclude that treatment of melanocytic naevi whether acquired or congenital with classical fusiform excision along the relaxed skin tension lines remains an effective and time tested procedure. There are least chances of scarring provided the basic principles of skin cosmesis are not violated.

\section{REFERENCES}

1. Anon. Consensus conference: Precursors to malignant melanoma. JAMA. 1984;251:1864-6.

2. Castilla E, Dutra M, Orioli-Parreiras I: Epidemiology of congenital pigmented naevi. Incidence rates and relative frequencies. $\mathrm{Br} \mathrm{J}$ Dermatol. 1981;104:307-15.

3. Zhu G, Duffy DL, Eldridge A, Grace M, Mayne C, O'Gorman L: A major quantitative-trait locus for mole density is linked to the familial melanoma gene CDKN2: a maximum- likelihood combined linkage and association analysis in twins and their sibs. Am J Hum Genet. 1999;65:483-92.

4. Wachsmuth RC, Turner F, Barrett JH, Gaut R, Randerson-Moor JA, Bishop DT: The effect of sun exposure in determining naevus density in UK adolescent twins. J Invest Dermatol. 2005;124:5662.

5. Menzies SW, Stevenson ML, Altamura D, Byth K: Variables predicting change in benign melanocytic naevi undergoing shortterm dermoscopic imaging. Arch Dermatol. 2011;147:655-9.

6. Kruk JJ, Lewandowicz E, Rykala: Surgical treatment of pigmented melanocytic naevi depending upon their size and location. J Acta Chir Plast. 1999;41:20-4.

7. Tursen U, Kaya TI, Ikizoglu G: Round excision of small, benign, papular and dome-shaped melanocytic naevi on the face. Int $\mathbf{J}$ Dermatol. 2004; 43:844-6.

8. August P.J, Ferguson J.E, Madan V: A Study of the Efficacy of Carbon Dioxide and Pigment-specific Lasers in the Treatment of Medium-sized Congenital Melanocytic Naevi. Br J Dermatol. 2011;16:1037-42.

9. Goldberg DJ, Zeichner JA, Hodulik SG: Q-switched laser irradiation of pigmented naevi: analysis of markers for malignant transformation. Lasers Med Sci. 2006;18:53.

10. Bhat W, Akhtar S, Akali A: Waste not, want not: technique to use redundant skin from elliptical skin lesion excision. Plast Reconstr Surg. 2010;126:140-1.

11. Kraft J, Murray CA: The fusiform skin excision: one recipe for success. J Cutan Med Surg. 2011;15:245-9.

12. Adams B, Levy R, Rademaker AE, Goldberg LH, Alam M. Frequency of use of suturing and repair techniques preferred by dermatologic surgeons. Dermatol Surg. 2006;32:682-9.

Copyright by Iffat Hassan, et al. This is an open access article distributed under the terms of the Creative Commons Attribution License, which permits unrestricted use, distribution, and reproduction in any medium, provided the original author and source are credited. 